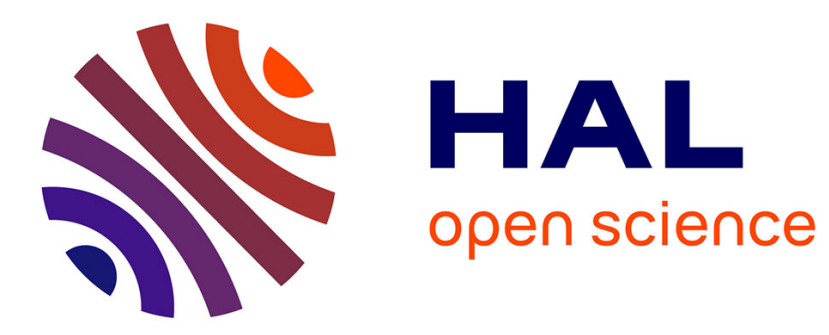

\title{
Sur la ligne de front de l'emploi.
}

Antoine Printz

\section{To cite this version:}

Antoine Printz. Sur la ligne de front de l'emploi.: Les politiques wallonnes d'accompagnement des demandeurs d'emploi dans l'isolement du bureau.. Émulations: Revue des jeunes chercheuses et chercheurs en sciences sociales, 2017. hal-01660855

\section{HAL Id: hal-01660855 https://hal.science/hal-01660855}

Submitted on 11 Dec 2017

HAL is a multi-disciplinary open access archive for the deposit and dissemination of scientific research documents, whether they are published or not. The documents may come from teaching and research institutions in France or abroad, or from public or private research centers.
L'archive ouverte pluridisciplinaire HAL, est destinée au dépôt et à la diffusion de documents scientifiques de niveau recherche, publiés ou non, émanant des établissements d'enseignement et de recherche français ou étrangers, des laboratoires publics ou privés. 


\section{Avertissement}

Le contenu de ce site relève de la législation belge sur la propriété intellectuelle et est la propriété exclusive de l'éditeur.

Les œuvres figurant sur ce site peuvent être consultées et reproduites sur un support papier ou numérique sous réserve qu'elles soient strictement réservées à un usage soit personnel, soit scientifique ou pédagogique excluant toute exploitation commerciale. La reproduction devra obligatoirement mentionner l'éditeur, le nom de la revue, l'auteur et la référence du document.

Toute autre reproduction est interdite sauf accord préalable de l'éditeur, en dehors des cas prévus par la législation en vigueur en Belgique.

\section{Référence électronique}

Citation : Antoine Printz, « Sur la ligne de front de l'emploi. Les politiques wallones d'accompagnement des demandeurs d'emploi dans l'isolement du bureau ", Émulations, en ligne. Mise en ligne le 10 décembre 2017. URL: http://www.revue-emulations.net/enligne/Printz-ligne-front-emploi-politiques-wallonnes

Éditeur : Émulations - Revue des jeunes chercheuses et chercheurs en sciences sociales http://www.revue-emulations.net 


\title{
Sur la ligne de front de l'emploi
}

Les politiques wallonnes d'accompagnement des demandeurs d'emploi dans l'isolement du bureau

\author{
Antoine Printz \\ Doctorant en sociologie à l'Université catholique de Louvain, Centre de \\ recherches interdisciplinaires Démocratie, Institutions, Subjectivité (CRIDIS)
}

\section{Résumé/Abstract}

[Fr] La littérature a conclu à l'avènement, au cours des dernières décennies, de l'État social actif. Dans le champ de la gestion du chômage, ce changement de référent a résulté, le 30 avril 2004, en un accord de coopération relatif à l'accompagnement et au suivi actif des chômeurs : le chômage sera désormais traité en Belgique, et donc en Wallonie, selon des modalités particulières, celles de l'accompagnement personnalisé du demandeur d'emploi. Les politiques d'emploi doivent être opérées au plus près de ce dernier, au travers de dispositifs d'action individuels et encadrés par le conseillerréférent, nouveau professionnel de l'emploi. L'enquête qui suit vise à mettre en lumière les conséquences d'une telle modification de l'action publique, qui repose dorénavant sur une relation établie entre l'agent mandaté et l'allocataire dans l'isolement du bureau. Au cours d'une enquête qualitative auprès de conseillers-référents (entretiens semi-directifs et observations) au sein de trois lieux de l'Office wallon de la formation professionnelle et de l'emploi (Le Forem), nous avons dégagé ce qui pourrait constituer les traits marquants de la pratique de tels professionnels qui, bien qu'étant des agents de l'État, sont très éloignés de l'idéal-type de la bureaucratie wébérienne. Cette évolution des pratiques professionnelles porte l'espoir d'une sortie des carcans dépersonnalisés et asphyxiants de l'action bureaucratique, mais comporte le risque de l'introduction dans le travail de la variable morale et d'une iniquité de traitement.

Mots clés : chômage, politiques d'emploi, activation, individualisation, accompagnement.

[En] During the last decades, literature has highlighted the emergence of the active welfare state. In the field of unemployment insurance, this shift resulted in the active support and supervision of job seekers. Unemployment is henceforth going to be dealt with under specific modalities in Belgium, hence in Wallonia. Those modalities are characterized by the personalized support of each job seeker. Employment policies must be carried out as close as possible to the job seeker, through supervised and personalized coaching by civil servants. The following research project will highlight the consequences of such a change regarding public action, which is now based on a close connection between state agents and beneficiaries, happening in the office's isolation. Through a qualitative sociological survey (interviews and observation) among employees within three Belgian public career offices, we managed to identify the potential significant features of the activities of such agents, that are very far removed from the classical Weberian bureaucracy. This change carries the hope of a getting out of the depersonalized and suffocating stranglehold of the bureaucratic action but also the risk of introducing a potential disparity of treatment and a moral variable in this new way of supporting the unemployed.

Keywords: unemployment, employment policies, activation, individualization, support. 


\section{Introduction}

Au cours des années 1990 et 2000, la gestion du chômage en Belgique s'est progressivement vue dotée de nouvelles structures et de nouvelles modalités d'action. Les demandeurs d'emploi ont été mis au centre de dispositifs nouveaux devant leur offrir «un accompagnement individuel pour décrocher un emploi » (Verhofstadt, 2003). Cette évolution participe d'un mouvement européen plus large qui vise à faire de l'État un agent actif contre le chômage, transformant le filet de sécurité de la protection sociale en un «tremplin pour l'emploi » (Blair, Schroeder, 1998 : 10). C'est l'avènement en Belgique de l'État social actif, censé rompre avec la passivité providentialiste en introduisant une conditionnalité accrue des droits, articulée au vœu de mise en place de dispositifs individualisés. L'objectif est de minimiser la dépendance aux prestations en relevant le défi face auquel l'État-providence a échoué : "améliorer la qualité de la protection sociale et inciter à effectuer la transition d'une allocation au travail » (Vandenbroucke, $2013: 25$ ). L'activation des prestations sociales, dans la lignée des prescriptions de la Stratégie européenne pour l'emploi, est consacrée par l'accord de coopération du 30 avril 2004. Cet accord entre l'État fédéral, les Régions et les Communautés affirme la volonté d'une prise en charge régionale coordonnée du chômage à l'aune du « droit à un accompagnement lié à l'obligation [de] collaborer de manière active [à sa remise à l'emploi $]^{1} »$.

C'est l'Office wallon de la formation professionnelle et de l'emploi (Le Forem) qui prend la charge de cette tâche nouvelle en Wallonie. Celle-ci est plus spécifiquement portée par le conseiller-référent, nouveau professionnel de l'emploi, qui s'oppose tant au placeur d'antan qu'au contrôleur d'aujourd'hui. Le conseiller-référent doit en effet dorénavant " accompagner» le demandeur d'emploi, en lui apportant un soutien direct en vue d'une recherche d'emploi optimale. Il dispose pour cela d'outils (aide directe, formation et expertise) mobilisés au cours d'entretiens en tête-à-tête d'une durée relativement conséquente, approchant souvent l'heure de temps, sur une période parfois longue et à intervalles réguliers.

Cet article a pour objectif d'éclairer les enjeux d'une telle délégation de l'action publique à la discrétion de ses agents d'exécution, en mobilisant l'ancrage théorique de la street-level bureaucracy, développé notamment par Michael Lipsky (1969; 2010), mais également par les travaux francophones d'ethnographie de l'action publique (Weller, 1990; $2000 ; 2010$; Dubois, 2012 ; 2014). Cette étude est issue d'une recherche empirique menée auprès de douze employés du Forem, officiant au siège provincial, dans un bureau de proximité ou dans une maison de l'emploi ${ }^{2}$, dont une part de l'activité professionnelle consiste ou a consisté en l'accompagnement de demandeurs d'emploi. Nous avons réalisé des entretiens compréhensifs avec ces conseillers, afin de tenter d'approcher la manière dont ces agents perçoivent leur travail d'accompagnement, ses enjeux, ses horizons et ses modalités. Ensuite, nous avons procédé à l'observation d'entretiens conduits par ces agents avec des demandeurs d'emploi afin de cerner comment cet accompagnement se mettait en place en pratique et selon quel rapport aux prescriptions politiques.

\footnotetext{
130 Avril 2004 - Accord de coopération entre l'État fédéral, les Régions et les Communautés relatif à l'accompagnement et au suivi actifs des chômeurs (Moniteur Belge du 25/07/2007, p. 39850).

${ }^{2}$ Le Forem est organisé selon quatre zones géographiques que sont le Hainaut, le Brabant wallon-Namur, LiègeHuy-Verviers et la province de Luxembourg. Chacune de ces zones dispose d'une direction régionale et d'un siège central qui constitue le centre névralgique de l'action territoriale d'insertion des demandeurs d'emploi sur le marché du travail et coordonne les activités sur la zone. Afin d'investir les territoires de manière optimale, Le Forem organise également ses activités au sein des bureaux de proximité (moyenne structure) et maisons de l'emploi, ou relais de l'emploi, qui sont de plus petites structures. En vue de préserver l'identité des conseillersréférents rencontrés dans le cadre de ce travail, et à la demande de la majorité d'entre eux, nous ne donnerons pas d'indications régionales plus avant et avons procédé à l'anonymisation des noms utilisés.
} 
Nous montrerons que le développement d'une action publique individualisée, marquée par le pouvoir discrétionnaire des conseillers-référents et éloignée des traits marquants de la pratique bureaucratique wébérienne a des conséquences ambivalentes, voire opposées. En effet, la délégation du pouvoir d'action à l'agent, marquée par l'impossibilité d'uniformisation des procédures, porte l'espoir d'une sortie des carcans administratifs. Ainsi, le développement d'interventions personnalisées et adaptées est rendu possible, alors qu'il ne l'était pas dans le cadre du traitement sur dossier. Cependant, cette personnalisation de l'action publique induit le risque d'une amplification de l'arbitraire des traitements, poussant vers une certaine iniquité. Le risque étant que les agents «à l'instar des philanthropes d'antan, [soient en position de] prendre des décisions fondées sur leurs perceptions subjectives plus que sur le droit ou une connaissance objective » (Dubois, $2012: 9$ ).

\section{Sur la ligne de front de l'emploi}

L'action publique de gestion du chômage, telle que conçue actuellement à travers l'accompagnement personnalisé, semble rompre avec les carcans classiques de la relation bureaucratique décrite par Max Weber. Il importe de noter que cette rupture n'est pas neuve. La gestion sociale du chômage a toujours mis en jeu des modes de fonctionnement relativement individualisés et peu formalisés ${ }^{3}$. Cependant, ceux-ci ont pris une ampleur non négligeable avec l'avènement du paradigme de l'accompagnement et du contrôle. En tant que domaine de l'action publique, la gestion sociale du chômage montre un éloignement patent vis-à-vis des traits significatifs de l'idéal-type bureaucratique wébérien, accru au cours des dernières décennies, dont il nous faudra mesurer les conséquences.

\subsection{Modèle wébérien et street-level bureaucracy}

L'organisation bureaucratique constitue un modèle de fonctionnement des États modernes occidentaux, en tant qu'elle est supposée garantir l'impartialité et l'équité de l'intervention politique (Della Porta, Mény, 1997). Elle est marquée par l'obéissance des fonctionnaires aux seuls «devoirs objectifs de leur fonction » et le formalisme comme «ligne de la moindre force » contre l'arbitraire (Weber, 1971: 223, 231). Ce formalisme bureaucratique permet l'avènement procédural d'un domaine public séparé du privé et l'impersonnalité de la relation qui y est mise en jeu (Aron, 1988).

La linéarité de la transmission entre prise de décision et mise en œuvre est ici centrale, en effet «les décisions fondamentales ne sont pas prises à l'intérieur de l'organisation bureaucratique, qui n'est qu'un système de transmission et d'exécution » (Touraine, 1959 : 10-11). Puisque ces agents se trouvent dans une position d'exécution, l'organisation bureaucratique se distingue "from other forms of social organizations in their adherence to formal hierarchy [...] and standard operating procedures » (Portillo, Rudes, 2014 : 322). Le fonctionnement bureaucratique met en jeu un ensemble de «traits de comportements et modèles de relation » qui assurent théoriquement une efficacité technique et l'impartialité visà-vis des usagers (Crozier, 1971 : 261).

Pourtant, certains pans de l'action publique semblent échapper à cette "élimination complète des relations personnelles et des considérations irrationnelles (affectivité, hostilité, anxiété, etc.) » (Merton, 1997: 190). Il s'agit de domaines dans lesquels les agents sont confrontés à la nécessité d'interagir avec l'usager et se voient dotés d'une grande discrétion dans leur action ; c'est par exemple le cas pour les personnels de l'enseignement, de police,

\footnotetext{
${ }^{3}$ On lira par exemple, pour s'en persuader, le Journal d'un chômeur de César Fauxbras (2014) qui donne à voir dans une écriture presque ethnographique le quotidien des chômeurs dans l'entre-deux-guerres parisien.
} 
des services sociaux (Lipsky, 1980). De la même façon, les agents du Forem sont constamment confrontés aux usagers - les demandeurs d'emploi - au cours d'interactions personnalisées en face-à-face. La conséquence de cette personnalisation semble avant tout être la visibilisation de la multiplicité des situations et l'impossibilité de procéduralisation des opérations.

\subsection{L'abstraction politique et l'épaisseur du terrain}

La « concrétude » du terrain est ressortie dès le début de la réalisation de notre enquête. Elle est souvent opposée à l'abstraction des discours venus du haut de la hiérarchie. Les agents du Forem, à l'inverse des politiques, endossent réellement la tâche d'accompagnement, étant à la front-line $e^{4}$ et devant parvenir à des interventions cohérentes, entre les règles imposées d'en haut et le street-level (Maynard-Moody, Leland, 2000 ; Maynard-Moody, Musheno, 2003). Ils se considèrent généralement comme en manque, voire en défaut, d'outils formalisés adaptés à la multiplicité des situations rencontrées sur le terrain et doivent dès lors adapter leur prestation de service selon l'individu présent et la relation mise en place avec lui. Le chômeur n'existe pas sur le terrain puisqu'il existe autant de chômeurs que de situations d'accompagnement : «[...] le problème qui se pose et que pose cet oisif malgré lui est tout différent selon qu'il s'agit d'un cadre ou d'un OS, d'un homme jeune ou de quelqu'un de licencié à quelques années de la retraite, d'une employée débutante ou d'une femme qui aurait voulu reprendre une activité après avoir élevé ses enfants »(Castel, 1991 : 138).

Le chômage est défini dans les arènes politiques comme un phénomène avant tout macrosocial. Dès lors, l'approche réductionniste ne peut être évitée et laisse l'agent d'exécution face à des situations non formalisées parmi lesquelles il doit trancher. Notre enquête a pu mettre en lumière la difficulté de la transmission entre les niveaux du politique et de l'intervention. La production réglementaire en politique est en effet considérée comme abstraite et déconnectée du terrain, ne cernant pas les réels enjeux et difficultés de sa réalité. La politique, comme «activité directive autonome» (Weber, 2005: 99), insuffle des mouvements d'action à ses agents selon une capacité d'abstraction jurant avec l'épaisseur de la réalité du travail social et administratif (Astier, 2000a). Les agents d'exécution sont confrontés aux données conditionnant leur travail, facteurs limitants ou déconnectés des contenus législatifs, nécessitant des logiques traductives et adaptatives. Il y a alors confrontation entre deux registres d'action, celui de l'élaboration de la politique et celui de sa mise en œuvre au niveau de l'interaction la plus réduite. Le premier est marqué par l'usage de catégories holistes et un horizon macrologique. Le registre de la mise en œuvre, à l'inverse, est caractérisé par des modalités plus ou moins empathiques, plus ou moins négociées, dès lors qu'il implique une mise en présence physique.

\section{3. «En chair et en os » : l'usager physiquement présent}

C'est en effet cette « incarnation physique » de l'usager qui éloigne le travail de l'agent du Forem de la simple mise en œuvre de cadres légaux, le contraignant à la prise en compte à la fois des nécessités intrinsèques à l'interaction et des caractéristiques particulières de l'usager. Cette mise en interaction des deux parties de la relation administrative implique de facto un engagement des interacteurs (Lipsky, 1969). Cela rend impossible le développement d'une perspective neutre et détachée, puisque l'accompagnement consacre une approche personnelle

\footnotetext{
${ }^{4}$ L'expression provient initialement de Lipsky (1969) et a nourri un ensemble de recherches, principalement anglo-saxonnes et scandinaves, associées au courant de la street-level bureaucracy. C'est partiellement dans ce courant théorique que s'inscrit cet article, y trouvant d'ailleurs son titre.
} 
et engagée ${ }^{5}$. En ce sens, Christine Charpentier et Maéva Duchène (2009) notent le caractère par essence «impliquant» de la relation d'accompagnement. Les conseillers rencontrés ne manquent pas de le pointer. Ils parlent systématiquement de «personne » et non de cas ou de dossier. Si cela peut sembler logique dès lors qu'ils se confrontent à des personnes, et non plus seulement à des dossiers, cela n'en est pas moins sans conséquence.

L'impossibilité, ou tout du moins la grande difficulté, d'abstraction ou de distance induit que la relation neutre n'existe pas et provoque une rupture avec l'idéal-type de l'administration bureaucratique, caractérisé par l'impersonnalité (Aron, 1988). Il s'agit en effet de ne pas négliger la situation d'entretien puisque celle-ci ne consiste pas en un échange neutre d'informations et d'instructions mais est une performance relationnelle située (Bartels, 2013). Dorénavant, et en rupture avec le modèle bureaucratique, l'usager fait une irruption physique dans le lieu de l'administration qui devient alors un «nouveau lieu d'arbitrage du droit » caractérisé par une relation renouvelée entre un agent, membre d'une " administration de responsabilité »- en opposition à une «administration de procédure »- et un usager personnalisé et incarné, qui n'est plus uniquement médié par le dossier (Weller, 2000 : 90). Apparaissent dès lors des variables inhabituelles dans la relation administrative classique.

\section{Empathie et compassion}

La relation de plus ou moins long terme entre le conseiller-référent et un demandeur d'emploi induit généralement le développement d'une attitude compréhensive de la part du premier, voire empathique, prenant le contre-pied de la stéréotypie dégradée du chômeur.

L'image chômeur-profiteur c'est complétement faux. La plupart des gens que je rencontre sont vraiment dans une recherche active d'emploi, sont demandeurs, sont volontaires. [...] Mais ça c'est ce qu'on voit, ceux qu'on ne voit pas, on ne sait pas. Mais ce que je sais c'est que la majorité de ceux qu'on voit sont vraiment dans une recherche active d'emploi et ils font leur job quoi. (Pierre, conseiller-référent dans un bureau de proximité)

Face à ce qu'ils considèrent comme une image stéréotypée du chômeur, démentie dans leur pratique professionnelle, il importe aux conseillers-référents de revaloriser celui-ci, en insistant justement sur le fait que «le » chômeur n'existe pas. Leur expérience du demandeur d'emploi est multiple et ne correspond que très rarement à celle qu'ils trouvent véhiculée dans les médias, le milieu politique ou leurs réseaux personnels.

L'image du chômeur véhiculée, ne fut-ce qu'en lisant les commentaires des gens sous les articles, et sociologiquement parlant, c'est un truc de fou ! Quand je vois ça, je me dis ben oui l'image du chômeur, moi je n'ai pas cette image là parce que... mon image à moi du chômeur, elle est multiple, elle est plurielle. Parce que je ne vois que ça à longueur de journée et c'est rare d'avoir vraiment deux fois le même profil quoi ! Donc je n'ai pas l'image du chômeur-profiteur, je n'ai pas l'image du chômeurglandeur. Il y en a, mais ce n'est pas... je n'ai pas cette image-là. (Véronique, responsable hiérarchique au siège provincial)

La condition de chômeur est souvent attachée symboliquement à une dépréciation individuelle (Eisenberg, Lazarsfeld, 1938 ; Paugam, 1991 ; 2013). Le conseiller-référent intervient donc dans un contexte d'instabilité statutaire - souvent accompagné de conditions sociales et économiques plutôt défavorables ou descendantes marquées par une certaine violence - dont il est même généralement l'instigateur. Le conseiller est en effet le premier interlocuteur de l'individu nouvellement chômeur, doté dès lors d'un fort pouvoir instituant. Il

\footnotetext{
${ }^{5}$ Prenons garde de ne pas associer cet engagement nécessaire à une posture de type militant ou idéologique : ici, la nécessité de l'engagement signifie uniquement l'impossibilité de mener à bien une interaction telle que celle sous-tendue par l'accompagnement personnalisé - longue, répétée et intime - sans un enrôlement impliquant une attitude compréhensive.
} 
est donc essentiel pour lui de ménager au maximum la face positive, déjà institutionnellement malmenée, de son interlocuteur ${ }^{6}$.

C'est très traumatisant pour certaines personnes. Moi j'en ai connu certains qui ne remettaient pas leur carte de chômage en fin de mois, donc ils se mettaient dans des situations de précarité financière parce que c'était trop pour eux d'aller remettre cette carte de chômage. C'était l'horreur absolue. La société ne se rend pas compte à quel point ça peut être difficile d'être au chômage ! Parce que ça coupe de tout. (Pierre, conseiller-référent dans un bureau de proximité)

Notons cependant que le développement de cette attitude compréhensive et empathique est souvent contrebalancé, de manière apparemment paradoxale, par une posture de suspicion visà-vis des allocataires, reprenant ici partiellement la méfiance à l'égard des demandeurs d'emploi, dans des termes et selon des modalités peu éloignées de celles qui renvoient au rejet de l'imaginaire social dégradé. Ainsi, il n'est pas rare de trouver dans le même entretien une attitude compréhensive à l'endroit de la difficulté économique de nombreux chômeurs suivie d'une accusation d'opportunisme et d'une caractérisation du chômeur comme consommateur d'allocations. C'est typiquement le cas dans nos entretiens lorsqu'est abordée la question du risque de radiation du demandeur d'emploi suite à une absence de réponse aux convocations par exemple. La réaction du demandeur d'emploi y est décrite bien loin des discours sur la multiplicité des profils et des postures compréhensives sur la violence intrinsèque aux dispositifs de traitement du chômage qui pourrait entraîner des absences. La sémantique utilisée est connotée négativement et marque l'inscription du demandeur d'emploi dans des catégories dépréciées : «en général quand les gens se rendent compte qu'il n'y a plus de sous qui arrivent sur le compte, là ils se réveillent et ils se demandent pourquoi ils ne reçoivent plus leur allocation », ou encore «l'organisme de paiement, généralement ça les gens voient à quoi ça sert ». Ce type de séquences assimile le demandeur d'emploi à un acteur en seule recherche d'indemnisation de son inactivité. Il en est ainsi dans cet extrait d'entretien :

Et ce qui est marrant, $\mathrm{j}$ 'utilise le terme marrant, c'est que à ce moment-là, ils se bougent et donc comme ils sont radiés, ils se réinscrivent comme demandeur d'emploi mais dans les $24 \mathrm{~h}$ ou $48 \mathrm{~h}$, et les deux autres courriers, et les tons des courriers c'est assez euh... pas violent mais... c'est lettre morte. Mais quand ils reçoivent le courrier comme quoi ils sont radiés, là ça se manifeste ! (Jeanne, conseillèreréférente en maison de l'emploi)

Cette lecture stéréotypée de certains comportements, outre qu'elle montre la saillance des stéréotypes, permet aux conseillers-référents d'élever leur seuil de résistance à l'apitoiement (Dubois, 2009). Stratégie de mise à distance, elle ouvre, dans ce cas par exemple, la possibilité de les identifier comme des consommateurs d'allocation plutôt que comme des allocataires dans une situation de forte précarité ne pouvant se permettre de perdre leur indemnité de chômage et recoupant ainsi le discours classique sur l'allocataire-profiteur.

Le rapport à l'usager est toutefois foncièrement personnalisé, voire personnel : contre le cadre réglementaire rigide, les agents portent une forte attention «to who their clients are, acting on their assessments of people's character and identity »(Bartels, 2013 : 473). Cette rupture avec la «froide» relation bureaucratique permet une prise en compte des variables

\footnotetext{
${ }^{6}$ En évitant toutefois de tomber, par des procédés de valorisation parfois infantilisants et relevant d'un domaine trop intime, dans une menace d' « incursion territoriale » (Kerbrat-Orecchioni, Traverso, $2007: 80$ ). Il importe d'éviter au maximum les «interventions paradoxales» qui, sous couvert de «redorer» la face positive de l'individu, ne constituent en vérité qu'une attaque pour sa face négative (Détrie, 1994 : 149). Nous avons pu assister à de telles séquences lors de nos observations directes d'entretiens où le conseiller-référent adoptait une attitude surérogatoire vis-à-vis d'achèvements et de réussites du demandeur d'emploi ; attitude qui bien que peut-être encourageante, voire gratifiante, comporte une forte violence symbolique de par la posture professorale qu'elle met en jeu, pour le moins inhabituelle dans les opérations interactionnelles classiques entre personnes adultes compétentes. De fait, certains compliments ou félicitations, dont le motif est sans aucun doute l'encouragement du demandeur d'emploi, sonnent faux dans nos sociétés marquées par une forte prétention à l'égalitarisme moral, lorsque ceux-ci draguent des contenus pouvant être considérés comme pontifiants, voire condescendants.
} 
individuelles et contextuelles compensatoire de la dépersonnalisation réglementaire ${ }^{7}$ tout en offrant une latitude plus grande dans les opérations administratives. Cependant, le développement d'une posture personnalisée comporte, dans le même temps, le risque de réintroduction de la morale dans le traitement des allocataires, et ce particulièrement dans un contexte de travail sous contrainte qui induit, comme nous l'avons montré, une nécessité d'arbitrage dans la qualité des prestations fournies.

\section{Pouvoir discrétionnaire et approche méritocratique}

Une seconde implication de la personnalisation du traitement de la question du chômage doit être envisagée. L'action publique à l'aune de l'accompagnement individuel permet la mise en place d'opérations où la reconnaissance individuelle prime, réintroduisant des variables relationnelles dans la «cage de fer » bureaucratique. Elle en porte tout du moins l'espoir. Cependant, le corolaire de l'empathie est le risque du développement de l'arbitraire et de l'iniquité. Lipsky $(1969$; 2010) a montré les stratégies adaptatives et le développement du pouvoir discrétionnaire dans ce type d'activité professionnelle, caractérisé par une limitation des ressources d'action (temporelles, théoriques et matérielles), idéalement nécessaires (Serre, 2005), comme par une relative absence de contrôle hiérarchique et une impossibilité marquée de procéduralisation du travail (Vinzant, Crothers, 1998).

Le pouvoir discrétionnaire des agents se développe sur une base évaluative morale, distinguant au sein des usagers ceux qui méritent l'intégralité des efforts de ceux qui méritent le «service minimum ». Face aux conditions et ressources restreintes apparaît le risque d'une discrimination en raison d'une inégalité de traitement. Lorsque les opérations administratives sont très peu formalisées et contrôlées ou encore marquées par de nombreux facteurs limitants, il peut donc être relativement fréquent de voir l'agent sélectionner parmi celles-ci, celles qui lui confèrent la plus grande satisfaction, le moindre désagrément selon une rationalité instrumentale ou même, selon une rationalité axiologique ici, celle qu'il juge la plus nécessaire ou méritée. Ainsi, les usagers jugés non coopératifs seront assez vite mis de côté, ceux qui ne rentrent pas dans le cadre imposé de la relation d'accompagnement également. Or, il n'est pas impossible que les critères d'évaluation morale soient du même ordre que ceux qui distinguent dans la logique institutionnelle et politique, le «bon chômeur », autonome et actif, du «mauvais chômeur », celui qui aurait justement le plus besoin d'un accompagnement dans sa recherche d'emploi, risquant alors d'accroître l'exclusion de ceux qui ne sont déjà pas « conformes ».

Pour avoir fait [occupé] plusieurs fonctions, je peux vous dire que les gens qui ont décidé de trouver un emploi, ils le trouvent, clairement. Ça dépend de la manière dont on s'y prend mais voilà, il y a toujours moyen de trouver un boulot. Je connais des gens qui ont vraiment envie de trouver un boulot mais ils n'ont pas de voiture, ils n'ont pas de permis, ils veulent trouver un boulot à deux milles euros à côté de chez eux, ben ils trouveront jamais ! Je pense que les gens qui s'y mettent, qui ont envie de chercher, de se former, d'évoluer, voilà, c'est la motivation qui compte. (Michel, responsable de l'inscription des demandeurs d'emploi au siège provincial)

Un «bon usager» sera donc privilégié dans un contexte où la prestation de service est contrainte et demande un certain engagement personnel de la part du conseiller. Celui-ci peut alors tendre à privilégier les accompagnements avec des demandeurs d'emploi qui, d'une part, lui apporte certaines gratifications et, d'autre part, sont conformes à son positionnement moral

\footnotetext{
${ }^{7}$ Souvenons-nous que selon ce modèle de fonctionnement, « le fonctionnaire [...] ne peut exercer son pouvoir vis-à-vis des assujettis que dans des limites très strictes; s'il les outrepasse, il est exposé à toutes sortes de recours gracieux et contentieux » (Boudon, Bourricaud, 2011 : 47).
} 
propre $^{8}$. Cette appréhension méritocratique de l'accompagnement est relativement prégnante dans le discours des conseillers, bien que non explicitée et assumée.

C'est l'archétype du chômeur! Avec son bonnet rasta, ses dreadlocks, qui sent l'ail, qui sent la bière, qui est tout le temps plein et qui n'est pas propre. Voilà. Lui il est toujours là. Mais un gars comme ça, ce n'est pas pour nous ça. C'est un cas psy ! Mais le problème, c'est que personne ne sait le prendre en charge ! En Flandre, ça aurait été vite fait bien fait transféré au CPAS ${ }^{9}$. Et la patate chaude serait au CPAS en disant «ok maintenant t'es SDF !». Mais ici, on ne peut pas. Maintenant, je ne suis pas $1^{\prime} O N E M^{10}$ non plus, je ne peux pas le priver de revenus hein! (Pierre, conseiller-référent dans un bureau de proximité)

Nous le voyons, certains usagers sont perçus comme ne relevant pas du travail d'accompagnement vers l'emploi, parce que trop éloignés de celui-ci. C'est dans ce cadre que se développe l'approche au mérite. Elle nous semble répondre à deux logiques : l'une instrumentale de minimisation de la charge de travail nécessaire et l'autre axiologique d'évaluation. L'extrait d'entretien suivant montre parfaitement la combinaison de ces deux logiques qui mènent au détachement vis-à-vis d'usagers peu enclins à entrer dans le « jeu » de l'accompagnement vers l'emploi selon les modalités valorisées - autonomie, comportement actif et loyauté à l'égard du conseiller - ainsi que, à l'inverse, à l'engagement personnel, voire à l'investissement affectif, dans les relations des conseillers avec les «bons » usagers, c'est-àdire les usagers conformes aux nécessités d'un accompagnement optimal.

Donc là j'ai un gars qui a mon âge. À mon âge n'avoir que le niveau primaire, l'obligation scolaire c'est jusqu'à dix-huit ans, tout son parcours professionnel était, il a toujours travaillé comme technicien de surface, tu te dis «Ouh là, ça va être dur quoi ». Et le dossier n'était pas complet, il n'avait pas de voiture, pas de permis, et donc pas de mobilité, ça va être dur quoi! C'est quasiment impossible à réinsérer sur le marché de l'emploi. Et on a des bonnes surprises parce que le gars il a pris une grosse baffe parce que ça faisait quatre CDD, il attendait son CDI et on lui a dit «non, tu coûtes trop cher !». Mais il a jamais... ce n'est pas comme quelqu'un qui a travaillé six mois et qui a glandé, et qui a chômé pendant des années ! Il a vraiment toujours travaillé et le gars je le vois ce matin, on remet à jour son dossier, il vient avec... il vient à l'heure !... Il vient avec un CV [Curriculum Vitae], il vient avec des outils qui sont bons. Il me dit « je suis déjà inscrit chez tel intérim, tel intérim ». Il fait tout quoi ! [...] On sort une offre d'emploi à Nivelles, il est parti déposer son CV et là il me téléphone pour me dire qu'il a déposé son CV ! Je sais bien qu'avec lui, il y a une confiance installée. Il avait besoin de vider son sac, je le laisse parler. Je n'embaume pas le paysage en disant que ça va être super facile mais je vais lui donner des pistes de réflexion, lui donner des trucs. C'est du bon sens quoi, ça n'a rien... mais ça remonte le moral des gens de se dire «voilà, j'ai quelqu'un qui m'accompagne, qui pense quand même à moi, qui va m'envoyer des offres ». Donc il sait qu'il peut compter sur moi pour l'accompagner dans ses démarches mais en attendant, le fait qu'il me téléphone pour me dire « voilà j'ai été là et $\mathrm{j}$ 'ai eu ça ! », ça me fait plaisir, parce que je sais que je peux compter sur lui. Il va faire les démarches qu'on a fixées. À l'inverse un jeune qui vient avec dix minutes de retard, il a abandonné l'école, enfin il a arrêté l'école pour faire l'armée, il vient sans CV et il me dit « je vous l'envoie demain ». C'était y a deux semaines! Ben un gars comme ça, je ne me casse pas le cul quoi! Parce qu'il joue avec mes pieds déjà ! Mais quand je dis je me casse pas le cul, ce n'est pas vrai dans le sens où je fais des démarches pour lui, je regarde avec lui pour s'inscrire pour l'armée, je fais en sorte qu'il rencontre des gens... Je fais des démarches vers lui, il prend ou il ne prend pas voilà quoi. J'attends toujours son CV, je ne crois pas l'avoir. Mais c'est la vie ça ! (Louis, conseiller-référent au siège provincial)

Nous pouvons voir dans cet extrait d'entretien l'inégalité de traitement opérée par les agents entre les usagers autant que l'investissement fort dans la relation : «ça me fait plaisir, parce

\footnotetext{
${ }^{8}$ Notons que certains travaux ont montré que le développement du comportement altruiste vis-à-vis de l'usager dépendait, entre autres, des affinités entretenues entre l'agent et son institution, le positionnant dès lors sur un continuum entre deux pôles de solidarité : politico-institutionnelle et d'équipe, avec le chômeur (Keiser, 2010 ; Buurman, Dur, 2012 ; Stensöta, 2012).

${ }^{9}$ Centre public d'action sociale : organisme public chargé de l'aide sociale, notamment via la dispense du revenu d'intégration sociale.

${ }^{10}$ Office national de l'emploi : institution fédérale en charge de l'organisation de l'assurance-chômage, du contrôle de disponibilité des demandeurs d'emploi (jusqu'à modification opérée par la sixième réforme de l'État en 2016) et des sanctions qui y sont attachées.
} 
que je sais que je peux compter sur lui ». À l'inverse par exemple du travail sur dossier qui peut être affectivement neutre, la relation d'accompagnement engage le conseiller. Cela comporte donc une possibilité de réintroduction de logiques évaluatives morales et de l'arbitraire des traitements, particulièrement accrue dans un contexte de faible contrôle hiérarchique et d'impossibilité d'uniformisation des procédures.

\section{Sauvetage et péril}

Comme nous l'avons vu, il importe d'insister sur le fait que le conseiller-référent peut être un agent d'atténuation des processus de dégradation statutaire, contrebalançant les injonctions dépersonnalisées par la mise en place de séquences interactionnelles compensatoires. La faculté d'écoute et d'empathie permet la réintroduction de la variable humaine dans le jeu institutionnel et administratif qui enserre l'usager. En ce sens, l'individualisation de l'accompagnement porte en elle le potentiel d'une ouverture sur un espace marqué par une certaine bienveillance, jurant avec la relation administrative classique. L'espace isolé du bureau constituerait le lieu d'une rupture dans le traitement dépersonnalisé de l'administration, par la création d'un lieu d'attention, marqué par le souci de prise en compte des contextes personnels et la volonté de combinaison «des principes d'égalité et d'universalité avec celui d'équité au regard des situations concrètes des personnes » (Astier, 2000c : 85). La personnalisation de l'accompagnement peut alors être vue comme palliatif des effets délétères des dispositifs classiques de protection sociale, récréant une certaine sociabilité dans un monde social qui en était relativement dénué.

Cependant, ces nouvelles modalités de gestion de la question du chômage ne sont pas dénuées de risques corolaires. Pour une fois, la formule de Friedrich Hölderlin peut être renversée ; permettons-nous de le faire au compte de notre conclusion : " là où croît ce qui sauve croît aussi le péril ${ }^{11}{ }$. Il est en effet un versant négatif à cette individualisation du traitement, celui de l'arbitraire et de l'évaluation méritocratique. Ainsi, les cadres uniformes et abstraits de la bureaucratie providentialiste, abhorrés il y a peu, en redeviendraient presque enviés : ceux-ci offraient la certitude d'un traitement uniforme basé sur la seule citoyenneté de l'individu. Ce n'est plus le cas. La singularisation des prestations de l'action publique mène au développement de «nouvelles magistratures du sujet » (Rosanvallon, 1995 : 211), introduisant le jugement de normalité dans leurs opérations, en dépit de la supposée ségrégation formelle entre la vie privée et l'administration publique. La variable morale est dorénavant partie prenante du travail de gestion du chômage et risque dès lors de transformer la prestation sociale en « une charité humiliante qu'on pourrait ou non concéder » (Gramsci, 2012 : 73), l'assimilant à ce « patronage inconscient et injurieux du riche aumônier » (Mauss, 2012 : 214). Il importe dès lors de garder ce risque à l'esprit afin de ne pas sombrer dans une dynamique préjudiciable pour l'État social et ses ayants-droit, porteuse du risque que le conseiller-référent, agent du Forem, ne devienne l'avatar de ce «juge des pauvres », qu'Alexis de Tocqueville, il y a près de 200 ans déjà, appelait de ses vœux.

\section{Bibliographie}

Aron R. (1988), Les étapes de la pensée sociologique. Montesquieu, Comte, Marx, Tocqueville, Durkheim, Pareto, Weber, Paris, Gallimard (« Tel»).

\footnotetext{
${ }^{11}$ Selon le vers de Friedrich Holderlin, poète romantique allemand, qui a écrit : « Mais là où croît le péril, croît aussi ce qui sauve. »
} 
Astier I. (2000a), «Weller Jean-Marc, L'État au guichet. Sociologie cognitive du travail et modernisation administrative des services publics, coll. "Sociologie économique", 1999 ; Dubois Vincent, La vie au guichet. Relation administrative et traitement de la misère, coll. "Études politiques", 1999. », Droit et Société, vol. 44, n 1, p. 253-260.

ASTIER I. (2000b), «Droit à l'emploi et magistrature sociale: vers une politique des situations ? », Droit et Société, vol. 44, n 1, p. 143-155.

AstiER I. (2000c), «Présentation du dossier "Les magistratures sociales" », Droit et Société, vol. $44, \mathrm{n}^{\circ} 1$, p. 85-89.

BARTELS K. P. R. (2013), «Public Encounters: the history and future of face-to-face contact between public professionals and citizens », Public Administration, vol. 91, n 2, p. 469-483.

BlaIR T., Schroeder G. (1998), Europe: The Third Way/Die Neue Mitte, En ligne, consulté le 10 juillet 2017. URL : http://library.fes.de/pdf-files/bueros/suedafrika/02828.pdf.

BOUDON R. (1999), « La "rationalité axiologique" : une notion essentielle pour l'analyse des phénomènes normatifs », Sociologie et sociétés, vol. 31, n 1, p. 103-117.

Boudon R., Bourricaud F. (2011), Dictionnaire critique de la sociologie, Paris, Presses universitaires de France.

BuURman M., DuR R. (2012), «Incentives and the Sorting of Altruistic Agents into StreetLevel Bureaucracies », The Scandinavian Journal of Economics, vol. 114, n 4, p. 1318-1345.

CASTEL R. (1991), «De l'indigence à l'exclusion, la désaffiliation. Précarité du travail et vulnérabilité relationnelle », in J. DONZELOT (dir.), Face à l'exclusion. Le modèle français, Paris, Éditions Esprit, p. 137-168.

ChARPentier C., DuchÈnE M. (2009), « L'accompagnement, un certain rapport des hommes entre eux... », Sciences-croisées, ${ }^{\circ} 5$. En ligne, consulté le 12 novembre 2017. URL: $\mathrm{http}: / /$ sciences-croisees.com/N5/charpentier.pdf.

CROZIER M. (1971), Le phénomène bureaucratique. Essai sur les tendances bureaucratiques des systèmes d'organisation modernes et sur leurs relations en France avec le système social et culturel, Paris, Seuil.

Della Porta D., MÉNy Y. (1997), Democracy and corruption in Europe, Social change in Western Europe, Londres, Washington, Pinter.

DÉTRIE C. (1994), «Lecture de: Les Interactions Verbales de C. Kerbrat-Orecchioni », Cahiers de praxématique, $\mathrm{n}^{\circ} 23$, p. 147-152.

DuboIs V. (2009), « Le paradoxe du contrôleur. Incertitude et contrainte institutionnelle dans le contrôle des assistés sociaux », Actes de la recherche en sciences sociales, vol. $3, \mathrm{n}^{\circ} 178$, p. 28-49.

Dubois V. (2012), «Ethnographier l'action publique. Les transformations de l'État social au prisme de l'enquête de terrain », Gouvernement et action publique, vol. 1, n 1, p. 83-101. 
Dubois V. (2014), «L'action de l'État, produit et enjeu des rapports entre espaces sociaux », Actes de la recherche en sciences sociales, vol. 1, $\mathrm{n}^{\circ}$ 201-202, p. 11-25.

EISENBERG P., LAZARSFELD P. F. (1938), «The psychological effects of unemployment », Psychological Bulletin, vol. 35, $\mathrm{n}^{\circ}$ 6, p. 358-390.

FAUXBRAS C. (2014), Viande à brûler. Journal d'un chômeur, Paris, Allia.

Gramsci A. (2012), Pourquoi je hais l'indifférence, Paris, Éditions Payot \& Rivages.

Kerbrat-Orecchioni C., Traverso V. (2007), Confidence dévoilement de soi dans l’interaction, Tübingen, M. Niemeyer Verlag.

KEISER L.R. (2010), «Understanding Street-Level Bureaucrats' Decision Making: Determining Eligibility in the Social Security Disability Program », Public Administration Review, vol. 70, $\mathrm{n}^{\circ}$ 2, p. 247-257.

LIPSKY M. (1969), «Toward a theory of street-level bureaucracy », Annual Meeting of the American Political Science Association, New York, The American Political Science Association.

LIPSKY M. (2010 [1980]), Street-level bureaucracy: dilemmas of the individual in public services, New York, Russell Sage Foundation.

MAuss M. (2012 [1925]), Essai sur le don. Forme et raison de l'échange dans les sociétés archaïques, Paris, Presses universitaires de France.

MAYNARD-Moody S., LELAND S. (2000), «Stories from the front-lines of public management: Street-Level workers as responsible actors », in J. L. BRUDNEY, L. J. O'TOOLE, H. G. RAINEY, Advancing public management: new developments in theory, methods, and practice, Washington, Georgetown University Press, p. 109-123.

Maynard-Moody S., Musheno M. C. (2003), Cops, teachers, counselors: stories from the front lines of public service, Ann Arbor, University of Michigan Press.

Merton R. (1997), Éléments de théorie et de méthode sociologique, Paris, Armand Colin.

PAUgAm S. (1991), «Les statuts de la pauvreté assistée », Revue française de sociologie, vol. $32, \mathrm{n}^{\circ} 1$, p. $75-101$.

PAUgAm S. (2013), La disqualification sociale essai sur la nouvelle pauvreté, Paris, Presses universitaires de France.

Portillo S., Rudes D. (2014), «Construction of Justice at the Street Level », Annual Review of Law and Social Science, vol. 10, $\mathrm{n}^{\circ} 1$, p. 321-334.

Rosanvallon P. (1995), La nouvelle question sociale. Repenser l'État-providence, Paris, Seuil.

SERRE D. (2005), "Gouverner le travail des assistantes sociales par le chiffre ? Les effets d'un indicateur informel », Informations sociales, vol. 5, n 167, p. 132-139. 
Stensöta H. O. (2012), « Political influence on Street-Level Bureaucratic Outcome: Testing the Interaction between Bureaucratic Ideology and Local Community Political Orientation », Journal of Public Administration Research and Theory, vol. 22, $\mathrm{n}^{\circ} 3$, p. 553-571.

Touraine A. (1959), «Situation du mouvement ouvrier », Arguments, vol. 3, n 12-13, p. 715 .

VANDENBROUCKE F. (2013), The active welfare state revisited, Bruges, die Keure.

VerhofstadT G. (2003), «Déclaration du gouvernement sur sa politique générale », Annales, $\mathrm{n}^{\circ} 3-13$, Sénat de Belgique.

VinZANT J. C., CROTHERs L. (1998), Street-level leadership: discretion and legitimacy in front-line public service, Washington, Georgetown University Press.

WEBER M. (1971), Économie et société, Paris, Plon.

WEBER M. (1991), Histoire économique. Esquisse d'une histoire universelle de l'économie et de la société, Paris, Gallimard.

WEBER M. (2005), Le savant et le politique, Paris, Éditions 10/18.

WELLER J.-M. (1990), « Sociologie d'une transaction : une caisse de retraités et ses usagers », Sociétés contemporaines, vol. $3, \mathrm{n}^{\circ} 1, \mathrm{p} .81-94$.

WeLLER J.-M. (2000), «Une controverse au guichet : vers une magistrature sociale ? », Droit et société, vol. 44, $\mathrm{n}^{\circ} 1$, p. 91-109.

Weller J.-M. (2003), «Le travail administratif, le droit et le principe de proximité », L’Année sociologique, vol. 53, $\mathrm{n}^{\circ}$ 2, p. 431-458.

Weller J.-M. (2010), «Comment les agents se soucient-ils des usagers ? », Informations sociales, vol. $2, \mathrm{n}^{\circ} 158$, p. 12-18. 